\title{
Time-Resolved ESR Study on Complex Radical Pairs Formed in the Photolysis of Methylene Blue Included in Water Soluble Sulfonated Calixarenes
}

\author{
Akiko Tanaka $^{1}$, Haruhiko Yashiro ${ }^{2,3}$, Asako Ishigaki ${ }^{4,5}$ and Hisao Murai ${ }^{5 *}$ \\ ${ }^{1}$ Department of Chemistry, Faculty of Science, Tohoku University, Sendai 980-8578, Japan \\ ${ }^{2}$ PRESTO, Japan Science and Technology Agency, Kawaguchi, Saitama 332-0012, Japan \\ ${ }^{3}$ Center for Quantum Science and Technology under Extreme Conditions, Osaka University, \\ Toyonaka, Osaka 560-8531, Japan \\ ${ }^{4}$ Japan Society for the Promotion of Science Fellow \\ ${ }^{5}$ Department of Chemistry, Faculty of Science, Shizuoka University, Shizuoka 422-8529, Japan
}

\begin{abstract}
The photochemical reactions of methylene blue (MB) included in water soluble sulfonated calix[n]arenes $(n=4,6,8)$ are studied using a time-resolved ESR method. The CIDEP (chemically induced dynamic electron polarization) spectra show the formation of the complex radical pair composed of the MB monocation radical and calixarene (phenoxyl-type) radical. The lifetime and broadened spectral shape are dependent on the size of the calixarene and are due to the longitudinal and transverse relaxation mainly induced by the tumbling motion of the radical pair with the spin dipole-dipole interaction. The pair dissociates in a few hundreds of nanoseconds in cases of $n=6$ and 8 .
\end{abstract}

* To whom correspondence should be addressed. Fax: +81-54-238-4753; E-mail: shmurai@ipc.shizuoka.ac.jp 


\section{Introduction}

Host molecules of inclusion compounds such as cyclodextrines (CDs) and calixarenes are attractive to many different fields because of their specific properties of formation of host-guest complexes.$^{1-6} \quad$ These specific inclusion properties can be used for the purpose of molecular identification and separation. The host-guest interaction in the complex is also known as a simplified model of biological enzymes. For photochemists, these inclusion conditions are potential reaction fields for special photocatalytic reactions. Especially, the formation of a transient paramagnetic intermediate known as a radical pair (RP) in the nano-sized confined space, which is the pathway for the consecutive reactions, is an attractive subject from the photochemical and spin-chemical point of view.

There were a few time-resolved ESR (tr-ESR) observations of photochemical reaction in confined conditions such as micellar and inclusion systems at low temperature. One of the reported inclusion systems dealt with the photoinduced electron transfer reaction in a $\gamma$-CD at low temperature. ${ }^{7}$ At low temperature, observation of such RPs trapped in organic or water glasses is rather easier since the intermediate radical species are stabilized keeping their distance close. However the observation of anisotropic interactions such as the magnetic dipole-dipole interaction which provides the structural information is possible at low temperature, the dynamic motion which contributes to the physical properties and the chemical reaction of the RP is frozen. The direct detection of the RP in confined conditions at room temperature using a tr-ESR may provide the interesting phenomena, such as the dynamic motion and the spin effect upon the chemical reaction. In case of micellar system, direct observation of RPs which provides the information of the exchange interaction is well known $^{8-11}$. The examples of CD system at room temperature were photolysis of benzoin in CD and photolysis of naphthoquinones in CDs in liquid phase. ${ }^{12,13}$ The former system did not show the RP spectrum but showed the ejection of the photo-decomposed fragment radicals from the cavity immediately after photolysis. The latter showed the hydrogen-atom 
abstraction reaction of the photoexcited triplet state naphthoquinones from the $\mathrm{C}-\mathrm{H}$ bonds of the cavity of $\alpha$-, $\beta$ - and $\gamma$-CDs followed by the ejection of semiquinone radicals from the cavity. In this case an unidentified broadened spectrum appeared at very early time region in the system of $\beta$-CD. This might be a confined RP in the CD cavity observed transiently at room temperature.

Calixarenes are composed of aromatic rings with phenolic hydroxyl groups and have ring-like structures which work as the host of inclusion compounds. Several calixarenes with different sizes are available. We can also modify them by introducing many different groups at the phenoxyl group and/or at the aromatic rings.

In the present paper, the photochemical reaction of methylene blue included in water soluble sulfonated calixarenes with different sizes is reported by means of a tr-ESR method at room temperature. The formation of complex RPs is shown, and the stability, $\mathrm{pH}$ dependence and the dynamics of the RPs in the cavity are discussed.

\section{Experimental}

Guaranteed reagents of methylene blue trihydrate $\left(\mathrm{MB}^{+}\right)$(Wako Pure Chemical) and 4-hydroxybenzenesulfonic acid (HBS) (Wako Pure Chemical) were used as received. Commercially available water-soluble three kinds of sulfonated calix[n]arenes $(n=4,6,8)$ (Calix[n]) were supplied by Sugai Chemi and used as received, where n denotes the number of the phenol frame unit. The solvent water was purified by the distillation after processing by $\mathrm{KMnO}_{4}$. The concentrations of three Calix[n]s were fixed at $40 \mathrm{mM}\left(\mathrm{M}=\mathrm{mol} \mathrm{dm}^{-3}\right)$. The concentration of HBS was kept at $240 \mathrm{mM}$. The low concentration of $\mathrm{MB}^{+}(0.4 \mathrm{mM})$ was employed in the aqueous solution with Calix[n]s and that with HBS.

We used an X-band ESR spectrometer (BRUKER esp-380E) with a slight modification for tr-ESR observation. ${ }^{14,15}$ The instrumental response time was about $100 \mathrm{~ns}$. The wavelength of $678 \mathrm{~nm}$ from a dye-laser head combined with an excimer laser (Lumonics EX-600; XeCl 
$308 \mathrm{~nm} ; 10 \mathrm{~Hz}$ ) was used to excite the sample inside the ESR cavity. The sample was deoxygenated by bubbling with nitrogen gas and flowed through a flat quartz cell with $0.3 \mathrm{~mm}$ optical path length. All the experiments were carried out at room temperature (ca. 24 degree Celsius). For the measurements of optical absorption spectra, SHIMAZU UV-1600PC photometer was used.

\section{Results and Discussion}

\section{1) Inclusion of $\mathrm{MB}^{+}$in Calix[n]s}

The absorption spectra of three Calix[n]s observed by a photometer showed the band in the UV region. The $\mathrm{pH}$ dependence of the absorption spectrum of Calix[n]s showed the shift of the absorption band to the longer wavelength under high $\mathrm{pH}$ conditions because the hydroxyl groups easily lost its proton(s). Similar pH dependence of the wavelength shift was also observed in the aqueous solution of HBS. This suggests that $\mathrm{pH}$ condition alters the properties of Calix[n]s such as the shape and its flexibility. Preliminary studies of $\mathrm{MB}^{+}$by means of photometer showed that $\mathrm{MB}^{+}$easily made the dimer and trimer depending upon the concentration. The low concentration of $\mathrm{MB}^{+}(0.4 \mathrm{mM})$ was employed for all the systems used here to avoid the formation of the dimer and the trimer. The absorption spectrum of $\mathrm{MB}^{+}$showed a strong band in the visible region of around $665 \mathrm{~nm}$ and this band is mainly composed of the monomer of $\mathrm{MB}^{+}$. However, even using low concentration of $\mathrm{MB}^{+}$, the addition of Calix[n]s changed the spectrum of the $\mathrm{MB}^{+}$. The addition of low concentration of Calix[n]s made the dimer and trimer of $\mathrm{MB}^{+}$probably because of the attractive force of Calix[n]. By increasing the concentration of Calix[n], the bands by the dimer and trimer diminished. This suggests that most of the $\mathrm{MB}^{+}$molecules are included in Calix[n]s and isolated as a single molecule in the cavity of Calix[n]s. In case of $\mathrm{MB}^{+} / \mathrm{Calix}[\mathrm{n}]$ systems, the data of the NMR spectrum proved that $\mathrm{MB}^{+}$is included in the cavity [unpub. data]. Since the absorption band of Calix[n]s located below $320 \mathrm{~nm}$ and that of $\mathrm{MB}^{+}$did not change drastically 
by the $\mathrm{pH}$ condition, the wavelength of $678 \mathrm{~nm}$ from the dye laser was good for the exclusive excitation of one $\mathrm{MB}^{+}$molecule included in the cavity of Calix[n].

\section{2) Laser photolysis of $\mathrm{MB}^{+} / \mathrm{HBS}$ system}

In order to investigate the primary step of the photoreaction of $\mathrm{MB}^{+}$and Calix[n]s which are the phenol derivatives, the system of $\mathrm{MB}^{+} / \mathrm{HBS}$ is first employed. The tr-ESR spectra observed in this system at pH of 5.07 and 1.16 are shown in Figure 1. The well dissolved CIDEP spectrum having emissive spin polarization with a slight E/A (emission/absorption) distortion and a $g$-factor of 2.0044 grows up in $700 \mathrm{ns.} \mathrm{This} \mathrm{spectrum} \mathrm{is} \mathrm{assigned} \mathrm{to} \mathrm{the}$ phenoxyl-type neutral radical of HBS (HBS') where a hydroxyl hydrogen is abstracted. The hyperfine (HF) coupling constants of ortho- and metha- protons of this phenoxyl radical are $a\left(\mathrm{H}_{\text {ortho }}\right)=0.68 \mathrm{mT}$ and $a\left(\mathrm{H}_{\text {metha }}\right)=0.21 \mathrm{mT}$, respectively. Figure 1 also shows a broadened spectrum superposed on that of HBS: Under the conditions of $\mathrm{pH}$ of ca. 5, this can be assigned to the methylene blue monocation radical $\left(\mathrm{MBH}^{+}\right)$having a g-factor of $2.0059 . .^{16,17}$ The spin polarization pattern of these species was explained by the triplet mechanism (TM ) polarization due to the excited triplet state of $\mathrm{MB}^{+}\left({ }^{3} \mathrm{MB}^{+*}\right)$ and the radical pair mechanism (RPM) of the triplet precursor. ${ }^{18,19}$ The primary step must be the hydrogen atom abstraction reaction by ${ }^{3} \mathrm{MB}^{+*}$ as follows:

$$
{ }^{3} \mathrm{MB}^{+*}+\mathrm{HBS} \rightarrow \mathrm{MBH} \cdot{ }^{+}+\mathrm{HBS} .
$$

The line width of the center HF lines of the spectrum of HBS` was slightly narrower than the outer ones. This is a typical phenomenon of chemical exchange reaction and probably due to the hydrogen hopping, in other words, the radical center hopping between HBS` and the ground state of HBS in the aqueous solution. The $\mathrm{pH}$ dependence of this system showed the strongest spectrum at slightly acidic conditions of around pH $5 . \quad$ Under much lower and 
higher $\mathrm{pH}$ conditions, the intensity decreased because of the obstruction of the hydrogen-atom abstraction reaction due to the proton addition to $\mathrm{MB}^{+}\left(\mathrm{MBH}^{2+}\right)$ at lower $\mathrm{pH}$, and the increase of the deprotonated HBS at higher $\mathrm{pH}$. The inefficient reaction under higher $\mathrm{pH}$ condition implies that the electron transfer reaction from deprotonated HBS to the excited $\mathrm{MB}^{+}$is not possible or much slower than that of the hydrogen abstraction reaction. Furthermore, the broadened spectrum of $\mathrm{MBH}^{+\cdot}$ shifts to the higher field $(-\Delta g=0.0020)$ under the lower $\mathrm{pH}$ conditions, and the center nearly superposes on the spectrum of HBS` as shown by a dotted line in Figure 1. This is probably because of the protonated radical formation such as $\mathrm{MBH}_{2}{ }^{2+\cdot}$ at lower $\mathrm{pH}$.

According to the CIDEP data of the system of $\mathrm{MB}^{+} / \mathrm{HBS}$, the following points become apparent. i) The hydrogen abstraction reaction of the excited triplet state of $\mathrm{MB}^{+}$from the hydroxyl group of HBS takes place in the acidic aqueous phase. ii) The electron transfer reaction between the excited triplet state of $\mathrm{MB}^{+}$and $\mathrm{HBS}$ may be very slow or may not take place. iii) The radical center hopping reaction between HBS` and HBS takes place in the solution. iv) At lower $\mathrm{pH}$ condition, $\mathrm{MBH}_{2}{ }^{2+}$. having slightly smaller g-factor is formed. These results of $\mathrm{MB}^{+} / \mathrm{HBS}$ system are applicable to the data analysis and discussion of MB/Calix[n] systems.

\section{3) Laser photolysis of $\mathrm{MB}^{+} / \mathrm{Calix}[\mathrm{n}]$ systems}

In the photolysis of three $\mathrm{MB}^{+} /$Calix[n] systems in acidic conditions, broadened emissive CIDEP spectra grow up in 200 ns as shown in Figures 2-a, b, and Figure 3 where the spectrum observed in $\mathrm{MB}^{+} / \mathrm{HBS}$ is given for comparison. The line width and time dependence of these systems differ each other. The time profiles of the peak intensity are shown in Figure 4. The fast emissive signal rise of these systems comparing with that of the $\mathrm{MB}^{+} / \mathrm{HBS}$ system indicates that the reaction takes place in the inclusion environment. Especially, the line width observed immediately after photolysis becomes broader in larger Calix[n]s. 
In case of $\mathrm{MB}^{+} / \mathrm{Calix}[4]$, the intensity and whole spectral shape observed at $\mathrm{pH}$ of 2.43 remain much longer than a few $\mu$ s as shown in Figures 2a and 4. The decay rate of this signal is $2.5 \times 10^{5} \mathrm{~s}^{-1}$ or smaller. This spectrum seems to be composed of two radical species because of the slightly asymmetrical spectral shape. The comparison of the shape with that of $\mathrm{MB}^{+} / \mathrm{HMB}$ system observed in the $\mathrm{pH}$ condition of ca. 5 leads to the conclusion that this is the superposition of broadened spectra of Calix[4] radical (Calix[4] $)$ and $\mathrm{MBH}^{+*}$. The broadening of the line width is due to the transverse relaxation time $\left(T_{2}\right)$ caused by the interaction as the RP of the complex form. Calix[4]' is thought to be the phenoxyl type radical formed by the hydrogen-atom abstraction reaction by ${ }^{3} \mathrm{MB}^{+*}$ included in Calix[4] as follows:

$$
\left({ }^{3} \mathrm{MB}^{+*}+\text { Calix }[4]\right) \rightarrow\left(\mathrm{MBH} \cdot{ }^{+} \cdots \text { Calix }[4] \cdot\right)
$$

where the parenthesis shows the inclusion conditions. The asymmetric feature proves that the $\mathrm{MBH}^{+}$does not exist outside of Calix[4]. Because the $\mathrm{pH}$ condition in the homogeneous media is very acidic and the whole spectrum must be symmetrical by the conversion of $\mathrm{MBH}^{+\cdot}$ to $\mathrm{MBH}_{2}{ }^{2+\cdot}$ as mentioned in the system of $\mathrm{MB}^{+} / \mathrm{HBS}$. Therefore, this slightly asymmetric spectrum is due to the long-lived complex spin-correlated RP of Calix[4] and $\mathrm{MBH}^{+\cdot}$ with emissive TM polarization. Another evidence of the formation of the complex RP is no clear observation of the free induction decay using the pulse ESR mode. These data show that the cavity of Calix[4] radical has an adequate size and property to hold the counter radical.

At lower $\mathrm{pH}$ of 1.23 , the signal intensity slightly decreased like the system of $\mathrm{MB}^{+} / \mathrm{HBS}$, probably because of the block of the hydrogen abstraction reaction by attachment of a proton to $\mathrm{MB}^{+}$. At higher $\mathrm{pH}$ of 12, a very weak and apparently RPM type CIDEP spectrum of Calix[4] $]^{\circ}$ with the HF structure was observed as shown in Figure 5. At high pH, many hydroxyl groups of Calix[4] are deprotonated and the structure of Calix[4] may change. Under this condition, efficient inclusion of $\mathrm{MB}^{+}$is unlikely. Probably, slow direct hydrogen abstraction from a few $\mathrm{OH}$ groups of Calix[4] takes place. This spectrum might be assigned 
to the Calix[4] radical with a spectral structure of 'triplet of triplet'; namely, two sets of two equivalent protons. According to Figure 5, three sets of two equivalent protons, $\mathrm{H}_{\mathrm{a}}, \mathrm{H}_{\mathrm{b}}$ and $\mathrm{H}_{\mathrm{c}}$, are anticipated and the HF coupling constants are $0.67 \mathrm{mT}$, nearly zero and $0.23 \mathrm{mT}$, respectively. From these information we can easily determine the structure of Calix[4]: The $\mathrm{C}-\mathrm{H}_{\mathrm{b}}$ bond direction is probably nearly perpendicular to the $\pi$-orbital occupied by an unpaired electron. This result implies that Calix[n] should show its HF structure, unless complex RPs are formed. Contrary to this result of Calix[4] at higher $\mathrm{pH}$, no HF structures were observed in any other systems of Calix[n]s.

In case of $\mathrm{MB}^{+} / \mathrm{Calix}[6]$, a broadened spectrum appearing at early time becomes sharp a little at late time at $\mathrm{pH}$ of 2.7 as shown in Figures 2-b and 4. The time profile of the peak signal is analyzed by two first order kinetics, and $k_{\text {fast }}=8( \pm 3) \times 10^{6} \mathrm{~s}^{-1}$ and $k_{\text {slow }}=4( \pm 2) \times 10^{5}$ $\mathrm{s}^{-1}$ are obtained. The broad spectral shape observed between 100 and 150 ns after photolysis shows the line width of $\Delta H_{\mathrm{fwhm}} \sim 2.5 \mathrm{mT}$. The line width change by time is thought to be due to disappearance of the broad component and appearance of a new sharper spectrum showing no HF structures. The possible dynamic process may be the escape of the $\mathrm{MBH}^{+}$from the Calix[6]', and the free radicals show a little sharper line width $\left(\Delta H_{\mathrm{fwhm}} \sim 1.8 \mathrm{mT}\right)$. This is because the line width of the spectrum at late time is close to that of the enveloped HF structure of the spectrum observed in the system of $\mathrm{MB}^{+} / \mathrm{HMB}$ at low $\mathrm{pH}$ (see Figure 1). The escaping $\mathrm{MBH}^{+\cdot}$ may be converted to $\mathrm{MBH}_{2}{ }^{2+\bullet}$ by protonation in the low $\mathrm{pH}$ medium and whole spectral shape becomes symmetrical as mentioned before.

$$
\left({ }^{3} \mathrm{MB}^{+*}+\text { Calix [6] }\right) \rightarrow\left(\mathrm{MBH} \cdot^{+} \cdots \text { Calix[6] }\right) \stackrel{\text { escape }}{\longrightarrow} \stackrel{\mathrm{H}^{+}}{\longrightarrow} \mathrm{MBH}_{2}^{2+} \cdot+\mathrm{Calix}[6] \cdot .
$$

The interesting point of the spectrum at late time is no appearance of the HF structures of free Calix[6] $]^{\cdot}$ contrary to the system of $\mathrm{MB}^{+} / \mathrm{Calix}[4]$ in high $\mathrm{pH}$ condition where the HF structure of free Calix[4] is observed. At lower $\mathrm{pH}$, the signal intensity decreased, probably because of the block of the hydrogen abstraction reaction by attachment of a proton to $\mathrm{MB}^{+}$as 
explained before in the system of Calix[4] / $\mathrm{MB}^{+}$. At higher $\mathrm{pH}$, no signal was observed at all.

In case of $\mathrm{MB}^{+} /$Calix[8], a broadened spectrum quickly disappears and a new weak spectrum appears shortly at late time at $\mathrm{pH}$ of 2.7 as shown in Figures 3 and 4 . The peak signal is analyzed by two first order decay kinetics, and $k_{\text {fast }}=7( \pm 3) \times 10^{6} \mathrm{~s}^{-1}$ and $k_{\text {slow }}=6( \pm 2) \times 10^{5}$ $\mathrm{s}^{-1}$ are obtained. These rates are similar to those of Calix[6] case, but the peak intensity of the latter component is very weak as shown in Figure 4. This is probably due to the fast spin relaxation compared with the dissociate rate from the inclusion condition. The broad spectral shape observed between 100 and 150 ns after photolysis shows the line width of $\Delta H_{\text {fwhm }} \sim 4.5$

$\mathrm{mT}$. The behavior of this system is very similar to that of $\mathrm{MB}^{+} /$Calix[6] except for the weak signal of the free radicals. The spectral shape and the line width at late time is also very close to that observed in the system of $\mathrm{MB}^{+} / \mathrm{Calix}[6]$. Therefore, the reaction process is as follows:

$$
\left({ }^{3} \mathrm{MB}^{+*}+\text { Calix [8] }\right) \rightarrow\left(\mathrm{MBH} \cdot{ }^{+} \ldots \text { Calix [8] }\right) \stackrel{\text { escape }}{\longrightarrow} \stackrel{\mathrm{H}^{+}}{\longrightarrow} \mathrm{MBH}_{2}^{2+} \cdot+\text { Calix [8] }
$$

In the lower and higher $\mathrm{pH}$ conditions, the qualitative behavior of the spectral change was similar to that of $\mathrm{MB}^{+} / \mathrm{Calix}[6]$.

The systems of $\mathrm{MB}^{+} / \mathrm{Calix}[\mathrm{n}] \mathrm{s}$ showed very different spectra and phenomena from that observed in the case of $\mathrm{MB}^{+} / \mathrm{HBS}$. These results strongly suggest the transient formation of relatively long-lived complex RPs in the system of $\mathrm{MB}^{+} / \mathrm{Calix}[\mathrm{n}]$. The slow tumbling motion of the RP having a spin dipole-dipole interaction induces very effective transverse relaxation $\left(T_{2}\right)$ that makes the spectral shape broad while it makes the longitudinal relaxation time $\left(T_{1}\right)$ much longer.

\section{4) Discussion of the complex radical pair}

The photo reaction of $\mathrm{MB}^{+} / \mathrm{HBS}$ takes place in homogeneous solution and there appears no RP spectrum. The only information obtained is strong TM and weak RPM spectral patterns 
induced by the spin polarization of the excited triplet state of $\mathrm{MB}^{+}$and the RP interaction of $\mathrm{MBH}^{+} / \mathrm{HBS}^{*}$ at the initial reaction stage. In a complex RP case, we can expect to observe a spin-correlated radical pair (SCRP) $)^{9,10}$. In case of $\mathrm{MB}^{+} / \mathrm{Calix}[\mathrm{n}] \mathrm{s}$, the whole spectral shape was much broader than that observed in the system of $\mathrm{MB}^{+} / \mathrm{HBS}$. There are two interactions as a RP, the exchange and spin dipole-dipole interactions. The exchange interaction $(J)$ of the RP may split the spectral lines a little by the value of $2 J^{9,10}$, which may work to affect the spectral width depending upon the $J$ value. Since this value is thought to be much smaller than the whole spectral line width of the complex RP, this isotropic interaction may not drastically affect the whole spectral shape in the present case. The interaction of the spin dipole-dipole may not work directly to construct the spectral shape, because this anisotropic interaction is easily averaged nearly zero by the free rotation of the complex RP. One of the most interesting features of the SCRP is the effect of the relative motion of the two radicals upon the spin dynamics by way of the exchange and the spin dipole-dipole interactions. The fluctuation of the exchange interaction caused by repetitive radical re-encounter that induces so called S-T dephasing ${ }^{23-27}$, can not be expected in the system of a fixed complex structure. The tumbling motion of the spin dipole-dipole interaction may induce very effective relaxation effects on the spectral shape and the decay of the signal.

Here, we should take into account the important factors of the spin relaxation induced by the tumbling motion of the RP having the anisotropic HF interaction, Zeeman effect (anisotropic $g$-factor) and spin dipole-dipole interactions. In general, the magnitude of the anisotropic HF coupling constants and $g$-factors are small enough to broaden the whole spectral shape by way of the transverse relaxation, $T_{2}$. The contribution of the spin dipole-dipole interaction to the line width of the complex RP is thought to be predominant, when two spins locate closely in the inclusion conditions. The spin relaxation times, $T_{1}$ and $T_{2}$, induced by the spin dipole-dipole interaction and the tumbling motion are given below ${ }^{20}$ : 


$$
\begin{aligned}
& \frac{1}{T_{1}}=\frac{\Delta^{2}}{10}\left\{\frac{2 \tau_{c}}{1+\omega^{2} \tau_{c}^{2}}+\frac{8 \tau_{c}}{1+4 \omega^{2} \tau_{c}^{2}}\right\} \\
& \frac{1}{T_{2}}=\frac{\Delta^{2}}{20}\left\{6 \tau_{c}+\frac{10 \tau_{c}}{1+\omega^{2} \tau_{c}^{2}}+\frac{4 \tau_{c}}{1+4 \omega^{2} \tau_{c}^{2}}\right\}
\end{aligned}
$$

where $\Delta, \omega$ and $\tau_{\mathrm{c}}$ denote the spin dipole-dipole interaction term of the complex RP, the spin angular frequency under ESR conditions and the rotational correlation time, respectively. $\quad \Delta$ is related to the zero-field splitting parameter $D$ by the following equation:

$$
\Delta=\sqrt{\frac{2}{3}} \frac{D}{\hbar}
$$

According to these equations, $T_{1}$ is nearly proportional to $\tau_{c}$ of the complex RP when $\tau_{c}$ is long enough $\left(\tau_{c}>\omega^{-1}\right)$. Contrary to this relation, $T_{2}$ is inversely proportional to $\tau_{c}$. These relations make the $T_{1}$ and $T_{2}$ values very different under slow tumbling motion condition and rationalize the observed data.

Here, let us think over the case of $\mathrm{MB}^{+} / \mathrm{Calix}$ [4] where no drastic spectral change by time was observed except slow decay of the signal intensity. The slightly distorted spectrum suggests that this RP spectrum is composed of $\mathrm{MBH}^{+\cdot}$ and Calix[4] as mentioned before. The slightly broadened spectral shape may be due to its $T_{2}$ and/or the appearance of the weak exchange interaction ${ }^{21,22}$. This implies that the spin dipole-dipole interaction is not very large, probably this value is less than $4 \mathrm{mT}$ or close to that of anisotropic HF and Zeeman terms, and the line width was not broad enough to obscure the total asymmetric shape. The roughly estimated line width of this particular system was much less than $0.5 \mathrm{mT}\left(\Delta H_{\mathrm{fwhm}}\right)$ which gave the $T_{2}$ value of 20 ns or much longer. The long observation time of $4 \mu$ s indicates that the complex structure is retained long time and the $T_{1}$ is very long comparing with the other two Calix[n] systems, because of the small anisotropic matrix elements and the slow rotational correlation time (see lines c) in Figure 6). 
In cases of $\mathrm{MB}^{+} / \mathrm{Calix}[6]$ and $\mathrm{MB}^{+} / \mathrm{Calix}[8]$ systems, the spectrum with a broad line width appearing immediately after laser photolysis may be explained by much smaller $T_{2}$ due to the strong spin dipole-dipole interaction and their slow rotational correlation time. The order of the broadness of the spectra is $\mathrm{MB}^{+} / \mathrm{Calix}[8]>\mathrm{MB}^{+} / \mathrm{Calix}[6]$. This result and the line width of $\mathrm{MB}^{+} /$Calix[4] system suggest that the transverse relaxation time $T_{2}$ is much smaller in the larger complex system. The $T_{2}$ values are roughly estimated from the line width of the spectra obtained by time-integration between 100 and 150 ns, and are about 2 ns and 4 ns for $\mathrm{MB}^{+} / \mathrm{Calix}[8]$ and $\mathrm{MB}^{+} /$Calix[6], respectively. When the instrumental response time is taken into account, the real $T_{2}$ values must be smaller than these values. These values are very short comparing with that of Calix[4] system. The $T_{2}$ values are related to the size of the complex RP and the distance between two radical centers of the complex, namely, the rotational correlation time and the spin dipole-dipole interaction. In order to estimate the rotational correlation times, $\tau_{c}$, of these complex systems, the averaged radius $a$ of the Calix[n] is roughly estimated as $0.4 \mathrm{~nm}, 0.6 \mathrm{~nm}$ and $0.7 \mathrm{~nm}$ by averaging the half of three dimensional lengths of $\mathrm{MB}^{+} /$Calix [4], $\mathrm{MB}^{+} / \mathrm{Calix}[6]$ and $\mathrm{MB}^{+} / \mathrm{Calix}[8]$ systems, respectively. The rotational correlation time, $\tau_{\mathrm{c}}$, is estimated according to the Einstein's relation given below ${ }^{20}$ :

$$
\tau_{c}=\frac{4 \pi \eta a^{3}}{3 k T}
$$

where $\eta, k$ and $T$ are viscosity of water, Boltzmann constant and temperature, respectively. This relation provides the $\tau_{\mathrm{c}}$ of $7 \times 10^{-11} \mathrm{~s}, 2 \times 10^{-10} \mathrm{~s}$ and $4 \times 10^{-10} \mathrm{~s}$, respectively. The zero field splitting parameter $D$ for $\mathrm{MB}^{+} / \mathrm{Calix}[4], \mathrm{MB}^{+} / \mathrm{Calix}[6]$ and $\mathrm{MB}^{+} / \mathrm{Calix}[8]$ systems can be estimated as ca.11 mT (or much smaller), ca.14 mT and ca.15 mT, respectively, according to Equation (6) and $T_{2}$ for these systems. Here, it is apparent that the $D$ for $\mathrm{MB}^{+} / \mathrm{Calix}[4]$ system is overestimated. Now the average distance of two spins can be obtained assuming a point dipole approximation. The distance $(d)$ between two radical centers in the complex is 
approximately determined to be $d\left(\mathrm{MB}^{+} / \mathrm{Calix}[4]\right)>$ ca. $0.64 \mathrm{~nm}, d\left(\mathrm{MB}^{+} / \mathrm{Calix}[6]\right)=$ ca. 0.59 $\mathrm{nm}$ and $d\left(\mathrm{MB}^{+} /\right.$Calix[8] $)=$ca. $0.57 \mathrm{~nm}$, respectively. According to Equation 5, the $T_{1}$ values of latter two systems are estimated to be $5 \times 10^{-7} \mathrm{~s}$ and $7 \times 10^{-7} \mathrm{~s}$, respectively. These values are a few times longer than the observed decay curvatures of $\mathrm{MB}^{+} / \mathrm{Calix}[6]$ and $\mathrm{MB}^{+} / \mathrm{Calix}[8]$ systems. This is because the $T_{2}$ values used for the calculation of $\Delta$ are overestimated due to the neglect of the instrumental response function and the fast decay components include both $T_{1}^{-1}$ and the ejection rate from the cavity. Anyhow, the estimation of $T_{1}$ and $T_{2}$ values qualitatively explains the time evolution of these systems. However, the large $T_{1}$ value of $\mathrm{MB}^{+} /$Calix [4] system indicates that the $d\left(\mathrm{MB}^{+} /\right.$Calix[4]) must be $0.9 \mathrm{~nm}$ or longer, and the $D$ value is smaller than $4 \mathrm{mT}$. Accordingly, it is quite plausible that these broadened spectra are due to the transiently formed complex RP at room temperature, if we consider the sizes of present three Calix[n]s. The qualitative concept of the relation between the $\tau_{\mathrm{c}}$ and spin relaxation times of $T_{1}$ and $T_{2}$ is drawn in Figure 6, where the most probable parameters discussed are employed.

The sharpened spectra without its HF structure of free radicals observed at late time in the system of $\mathrm{MB}^{+} /$Calix[6] and $\mathrm{MB}^{+} /$Calix[8] may be due to Calix[n] radical and $\mathrm{MBH}_{2}{ }^{2+}$. superposed on it. The broadened shape of Calix[n] ${ }^{\circ}$ without their HF structure may be caused by the hopping of the phenoxyl-type radical center of free Calix[n] radicals around the OH groups. This is because the HF dependent line broadening is observed even in the system of $\mathrm{MB}^{+} / \mathrm{HBS}$ where inter molecular radical center hopping might occur. The hopping rate is roughly estimated by assuming that the broadening is mainly due to the hopping of hydrogen atom. The simulation trial to reproduce the width assuming a Lorentz line shape for every HF line of the Calix[n] at low $\mathrm{pH}$ condition gave the rate of $1 \times 10^{8} \mathrm{~s}^{-1}$. In case of $\mathrm{MB}^{+} / \mathrm{Calix}[4]$, the contribution of the hopping to the $T_{2}$ cannot be recognized because of the failure of the free radical observation in this system at low $\mathrm{pH}$ condition. At higher $\mathrm{pH}$, the appearance of the HF structure indicates that the hopping of the radical center is not fast enough because of the 
lack of hydroxyl hydrogen atoms.

\section{Conclusion}

The photochemical reactions of methylene blue included in water soluble sulfonated calix[n]arenes $(n=4,6,8)$ show the CIDEP spectra of the complex RP composed of the MB monocation radical and phenoxyl-type calixarene radicals. The data are compared with the photo-reaction of $\mathrm{MB}^{+}$and $\mathrm{HMB}$ in homogeneous medium. The decay time and broadened spectral shape are dependent on the size of the calixarene and are due to the longitudinal and transversal relaxation mainly induced by the tumbling motion of the RP with the spin dipole-dipole interaction. The pair dissociates in a few hundreds of nanoseconds in cases of $n$ $=6$ and 8 , but is stabilized as the complex pair in case of $n=4$. The free radicals of Calix[n]s does not show their HF structure, and this is due to the hopping of the radical center in the $\mathrm{OH}$ groups of Calix[n].

\section{Acknowledgment}

Authors would like to express their thanks to Prof. Hayashita at Tohoku University for his valuable suggestion and discussion about inclusion compounds. Authors are also grateful to Instrumental Analysis Center for Chemistry of Tohoku University for the observation of NMR spectra of the present system. A.I. is grateful to the grant of Japan Society for the Promotion of Science. 


\section{References}

[1] Bender, M. L., Komiyama, M.: Cyclodextrin Chemistry. Springer-Verlag, Berlin (1978)

[2] Reddy, G. D., Ramamurthy, V.: J. Org. Chem. 52, 3952-3956 (1987)

[3] Rao, B. N.; Syamala, M. S.; Turro, N. J.; Ramamurthy, V.: J. Org. Chem. 52, 5517-5521 (1987)

[4] Shinkai, S., Mori, S.: J. Am. Chem. Soc. 108, 2409-2416 (1986)

[5] Zhang, Y., Agbaria, R. A., Mukundan, N. E., Warner, I. M.: J. of Inclusion Phenomena and Molecular Recognition in Chemistry. 24, 353-365 (1996)

[6] Nishida, M., Ishii, D., Yoshida, I., Shinkai, S.: Bull. Chem. Soc. Jpn. 70, 2131-2140 (1997)

[7] Murai, H., Yamamoto, Y., I'Haya, Y. J.: Can. J. Chem., 69, 1643-1648 (1991)

[8] Sakaguchi, Y., Hayashi, H., Murai, H., I'Haya, Y. J.: Chem. Phys. Letters. 110, 275-279 (1984)

[9] Ishiwata, N., Murai, H., Kuwata, K., Sakaguchi, Y., Hayashi, H.: Z. Phys. Chem. 182, 41-50 (1993)

[10] Ishiwata, N., Murai, H., Kuwata, K.: Bull. Chem. Soc. Jpn. 68, 1315-1322 (1995)

[11] Ishiwata, N., Murai, H., Kuwata, K.: Res. Chem. Intermed. 19, 59-66 (1993)

[12] Kitahama, Y., Murai, H.: Chem. Phys. Letters. 261, 160-164 (1996)

[13] Takamori, D., Aoki, T., Yashiro, H., Murai, H.: J. Phys. Chem. (A). 105, 6001-6007 (2001)

[14] Fukuju, T., Yashiro, H., Maeda, K., Murai, H., Azumi, T.: J. Phys. Chem. (A), 101, 7783-7786 (1997)

[15] Fukuju, T., Yashiro, H., Maeda, K., Murai, H.: Chem. Phys. Letters. 304, 173-179 (1999)

[16] Braswell, E.: J. Phys. Chem., 72, 2477-2483 (1968)

[17] Contineau, M., Iliescu, C., Ciureanu, M.: Radiochem. Radioanal. Letter, 57, 9 (1983)

[18] Muus, L. T., Atkins, P. W., McLauchlan, K. A., Pedersen, J. B.: (eds.), Chemically Induced Magnetic Polarization. D. Reidel, Dordrecht (1977) 
[19] K. A. McLauchlan, in A. J. Hoff, (ed.), Advanced EPR, pp. 345-369, Elsevier, Amsterdam (1989)

[20] Carrington, A., McLachlan, A. D.: Introduction to Magnetic Resonance, Harper, New York (1967)

[21] Buckley, C. D., Hunter, D. A., Hore, P. J., McLauchlan, K. A.: Chem. Phys. Lett. 135, 307-312 (1987)

[22] Closs, G. L., Forbes, M. D. E., Norris, J. R.: J. Phys. Chem. 91, 3592-3599 (1987)

[23] Shushin, A. I.: Chem. Phys. Lett. 181, 274-278 (1991)

[24] Salikhov, K. M.: Appl. Magn. Reson. 13, 415-437 (1997)

[25] Salikhov, K. M., Sakaguchi, Y., Hayashi, H.: Chem. Phys. 220, 355-371 (1997)

[26] Sekihara, A. H., Honma, T., Fukuju, K., Maeda, K., Murai, H.: Res. Chem. Intermed. 24, 859-877 (1998)

[27] Tarasov, V. F., Yashiro, H., Maeda, K., Azumi, T., Shkrob, I. A.: Chem. Phys. 226, 253-269 (1998) 


\section{Figure captions}

Figure 1. Time-resolved ESR spectra observed in the system of $\mathrm{MB}^{+} / \mathrm{HBS}$ at $\mathrm{pH}$ of a) 5.1 (solid line) and b) 1.2 (dotted line).

Figure 2. Time-resolved ESR spectra observed in the system of a) $\mathrm{MB}^{+} / \mathrm{Calix}[4]$ at $\mathrm{pH}$ of 2.4, b) $\mathrm{MB}^{+} / \mathrm{Calix}[6]$ at $\mathrm{pH}$ of 2.7. The spectra observed in the system of $\mathrm{MB}^{+} / \mathrm{HBS}$ at $\mathrm{pH}$ of 5.1 are shown for comparison.

Figure 3. Time-resolved ESR spectra observed in the system of $\mathrm{MB}^{+} / \mathrm{Calix}[8]$ at $\mathrm{pH}$ of 2.7. The spectra observed in the system of $\mathrm{MB}^{+} / \mathrm{HBS}$ at $\mathrm{pH}$ of 5.1 are shown for comparison.

Figure 4. Time-dependence of the signal intensity of the center part of three tr-ESR spectra observed in the system of $\mathrm{MB}^{+} / \mathrm{Calix}[\mathrm{n}] \mathrm{s}$.

Figure 5. Time-resolved ESR spectra observed in the photolysis of $\mathrm{MB}^{+}$/ $\mathrm{HBS}$ system at $\mathrm{pH}$ of 12. The inset shows the structure of Calix[4] radical and its stick spectrum.

Figure 6. The conceptual diagram of the relation between $\tau_{\mathrm{c}}$ and spin relaxation times of $T_{1}$ and $T_{2}$. Spin relaxation curvatures estimated by the line width observed and $\tau_{\mathrm{c}}$ in the system of a) $\mathrm{MB}^{+} /$Calix[8] and b) $\mathrm{MB}^{+} /$Calix[6] where the values of a) are overestimated. c) The curvatures estimated by the line width and large $T_{1}$ value of $\mathrm{MB}^{+}$/ Calix[4] system as the minimum value (see text). 


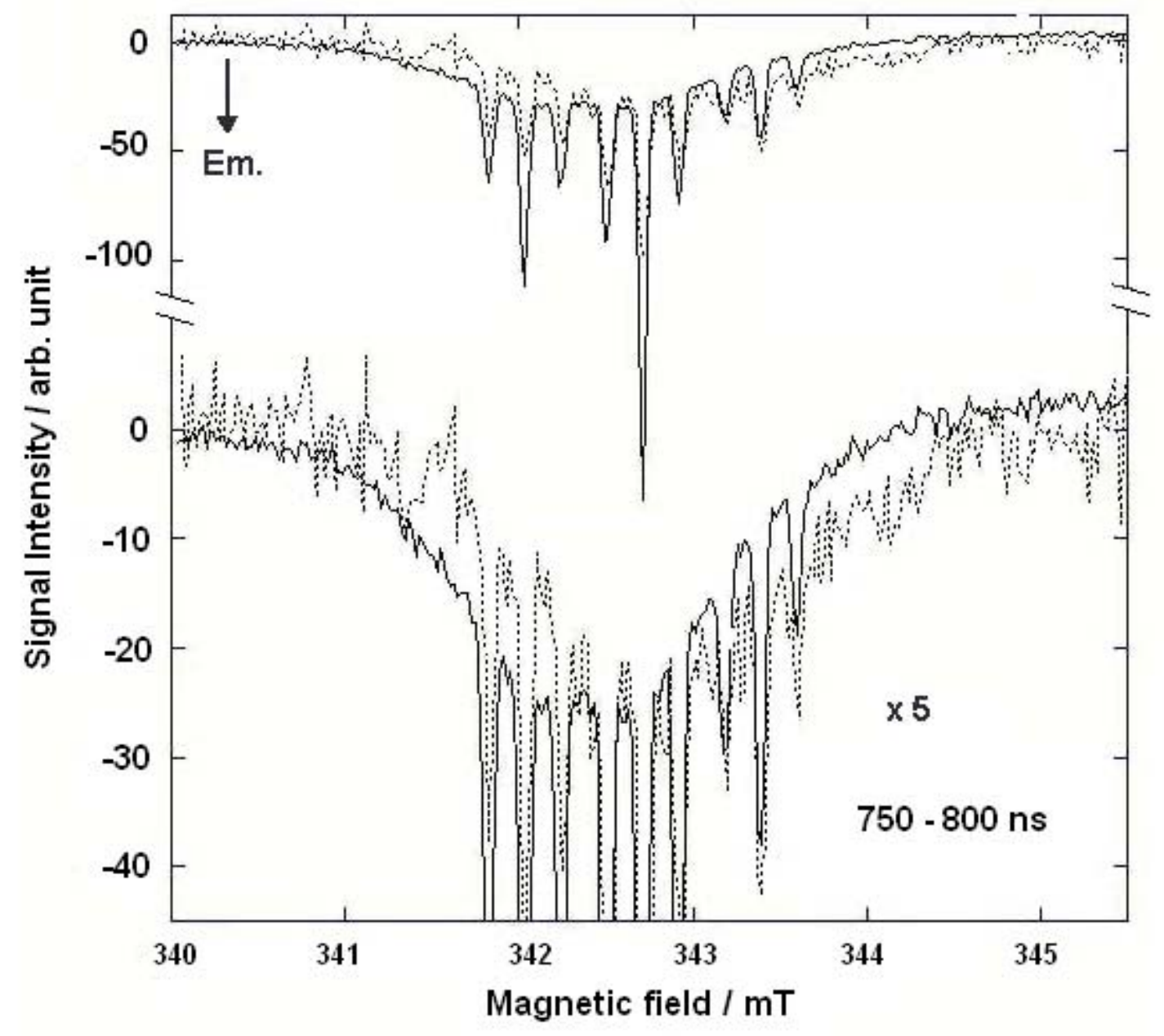

Fig. 1. A. Tanaka, et al. 
a)

b)

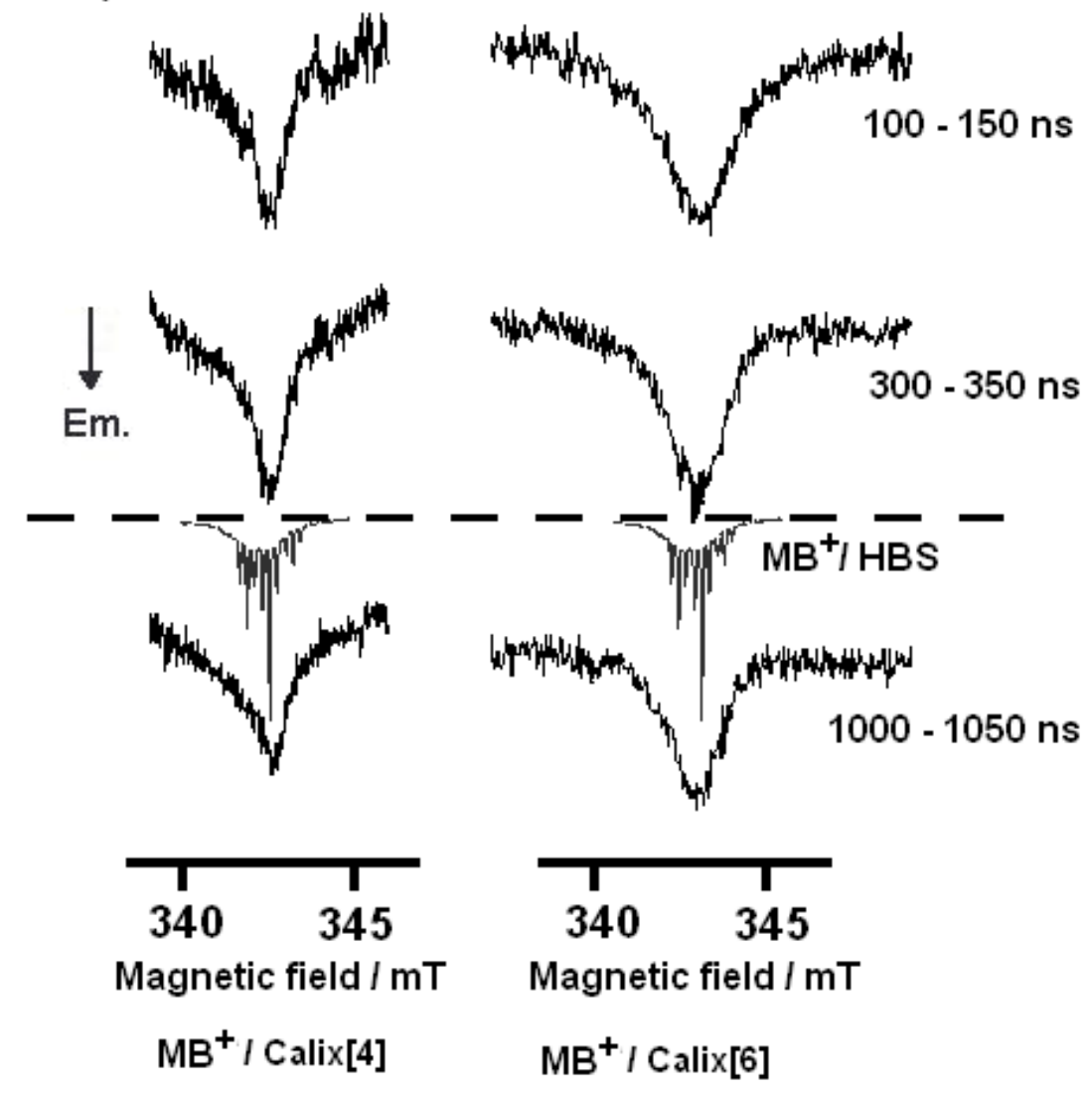

Fig. 2. A. Tanaka, et al. 

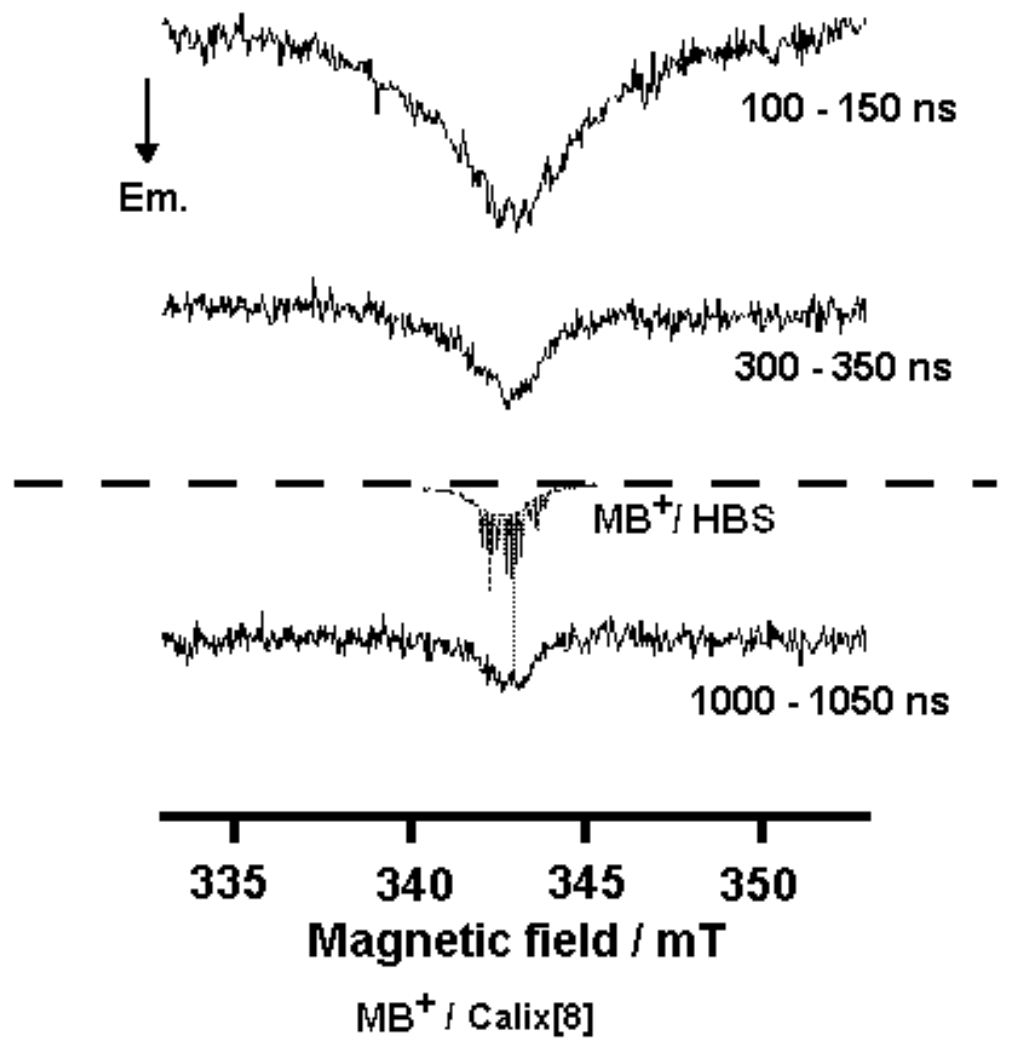

Fig. 3. A. Tanaka, et al. 


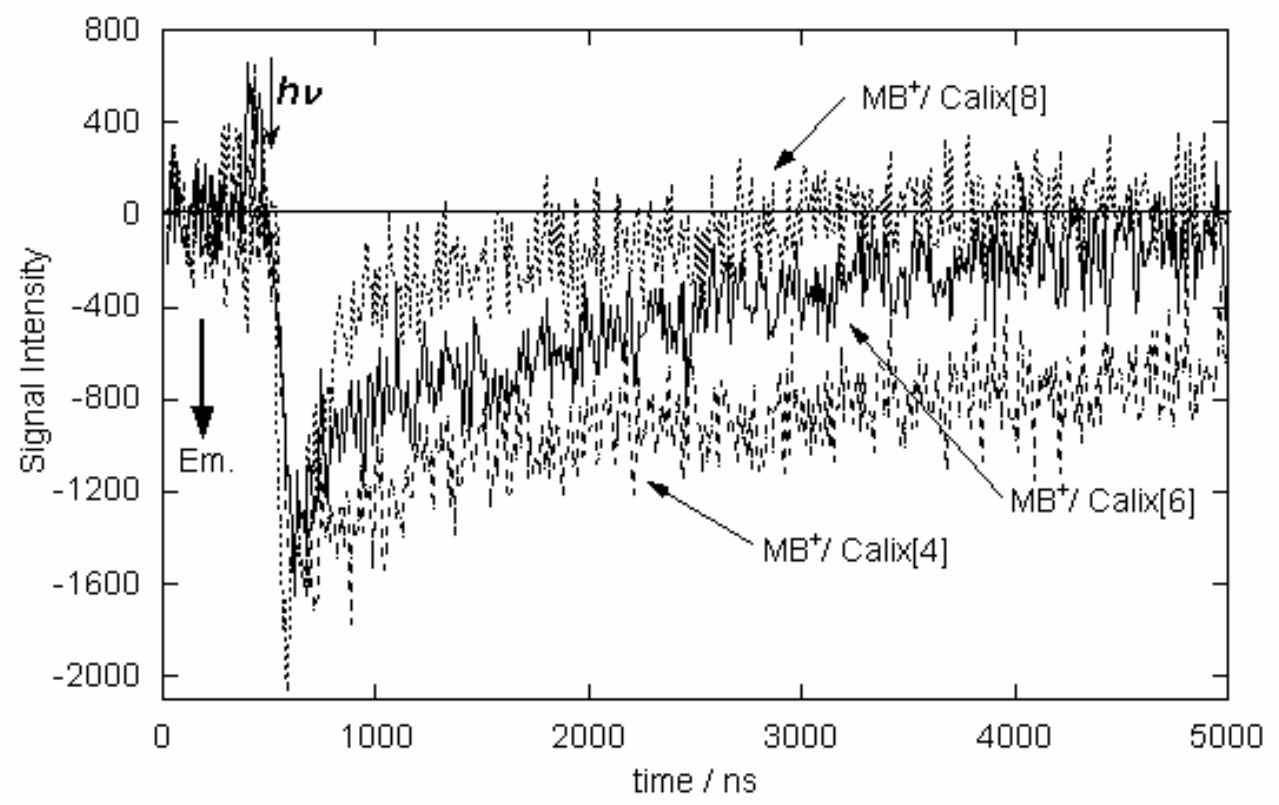

Fig.4 A. Tanaka, et al. 


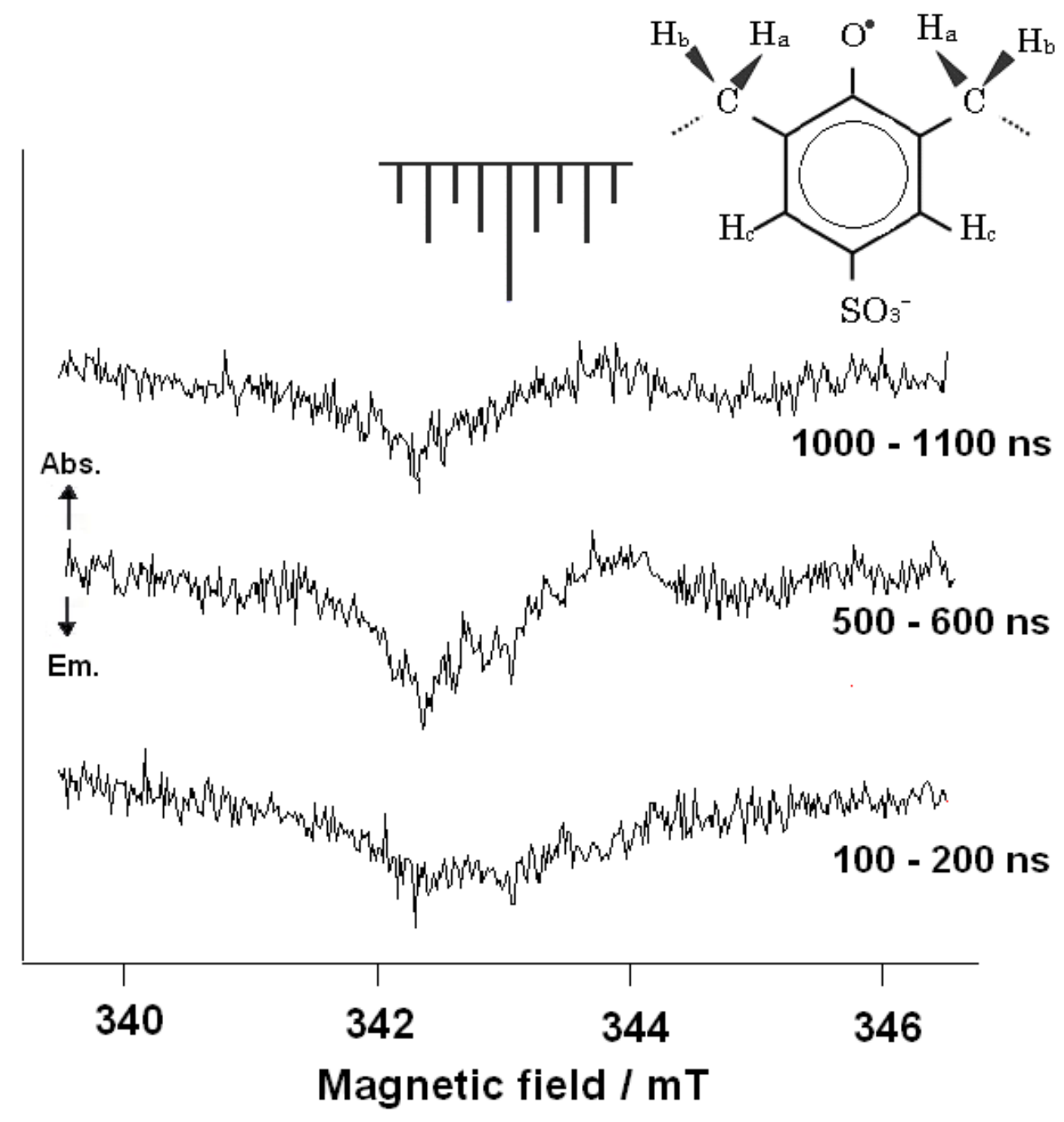

Fig. 5. A. Tanaka, et al. 


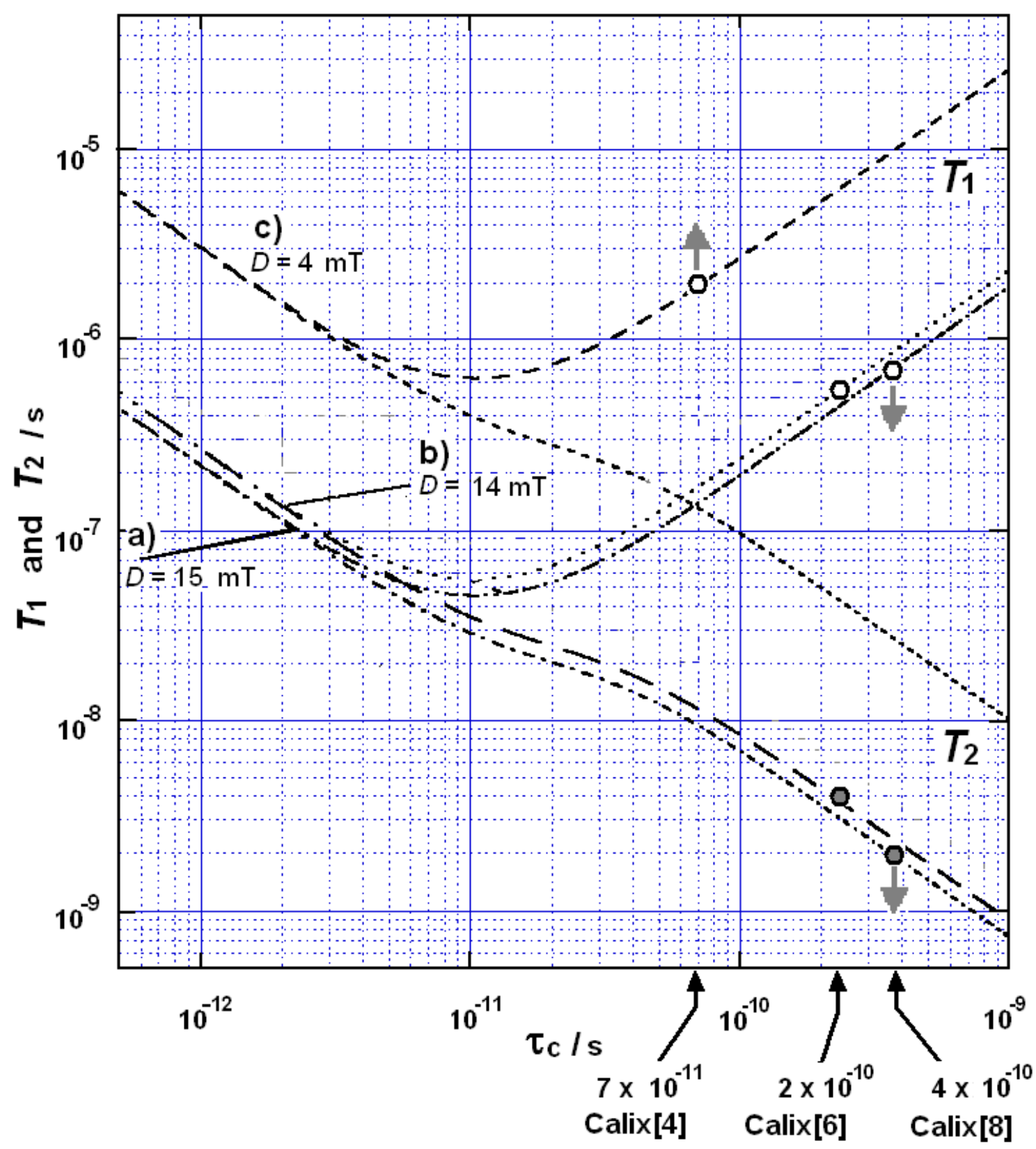

Fig. 6. A. Tanaka, et al. 